\title{
Apoyo familiar en la adherencia al tratamiento de pacientes con tuberculosis
}

\section{Family support in the adherence to the treatment of patients with tuberculosis}

\section{Suporte familiar na adesão ao tratamento de pacientes com tuberculose}

María A. Cedeño-Ugalde

maria.cedeñou@uleam.edu.ec

Fátima M. Figueroa-Cañarte

fatima.figueroc@uleam.edu.ec

Jonathan R. Zambrano-Barre

janatha1990@hotmail.com

Claudia V. Romero-Castro

romero.clau@yahoo.es

Carmen V. Arias-Nazareno

arias-carmen78@hotmail.com

Elio A. Santos-Andrade

santos02000@gmail.com

Recibido: 27 de junio de 2018 * Corregido: 28 de agosto de 2018 * Aceptado: 20 de septiembre de 2018

I. Doctora en Medicina y Cirugía; Magister en Epidemiologia; Docente Carrera de Medicina Facultad de Ciencias Médicas Universidad Laica Eloy Alfaro de Manabí, Manta, Ecuador.

II. Licenciada en Enfermería; Magister Gerencia en Salud para el Desarrollo local; Docente de la Universidad Laica Eloy Alfaro de Manabí, Manta, Ecuador.

III. Médico Cirujano en Práctica Privada, Universidad Laica Eloy Alfaro de Manabí, Manta, Ecuador.

IV. Médico Cirujano; Ministerio de Salud Pública, Quito, Ecuador.

v. Médico Cirujano; Ministerio de Salud Pública, Quito, Ecuador.

VI. Doctor; Seguro Campesino El Carmen Porvenir, Manabí, Ecuador. 


\section{Resumen}

La tuberculosis (TB) es una enfermedad triste que ha causado mucho daño a lo largo de la historia, afectando principalmente a las poblaciones más pobres y constituyendo uno de los mayores retos en salud pública actualmente. Aún con los avances globales en desarrollo y en las ciencias médicas desconcierta que siga siendo una enfermedad tan prevalente al comienzo de este siglo, con una tercera parte de la población mundial infectada y con 2 millones de personas fallecidas al año, declarada así como una urgencia mundial y enfrentando un obstáculo muy importante para su control: el abandono del tratamiento también llamado no adherencia, incumplimiento o falta de apego del paciente a la medicación que implica graves consecuencias tanto para el enfermo, como para la comunidad, debido a que el paciente no se cura y continúa la cadena de transmisión de la enfermedad. En el presente estudio se abordan aspectos teóricos de importancia que han contribuido a la fundamentación del tema; se ha organizado en base a los enfoques que incluye los conceptos de apoyo familiar y la adherencia al tratamiento con tuberculosis pulmonar.

Palabras claves: Tuberculosis Pulmonar; Paciente; Tratamiento; No Adherencia; Apoyo Familiar. 


\begin{abstract}
Tuberculosis (TB) is a sad disease that has caused a lot of damage throughout history, affecting mainly the poorest populations and constituting one of the greatest challenges in public health today. Even with the global advances in development and in the medical sciences, it is puzzling that it continues to be a disease so prevalent at the beginning of this century, with a third of the world population infected and with 2 million people dead every year, declared as an emergency world and facing a very important obstacle to its control: the abandonment of treatment also called nonadherence, non-compliance or lack of patient attachment to medication that implies serious consequences for both the patient and the community, because the patient does not the chain of transmission of the disease is cured and continues. In the present study important theoretical aspects that have contributed to the foundation of the subject are addressed; It has been organized based on approaches that include the concepts of family support and adherence to treatment with pulmonary tuberculosis.
\end{abstract}

Key words: Pulmonary Tuberculosis; Patient; Treatment; Non-Adherence; Family Support. 


\section{Introducción.}

La Tuberculosis es un problema de salud pública, no solamente en el país sino también a nivel mundial, de acuerdo a los datos estadísticos de la OMS es la novena causa de muerte y la primera por enfermedades infecciosas. En 2016 se notificaron 6,3 millones de nuevos casos de TB (frente a los 6,1 millones de 2015), lo que equivale al 61\% de la incidencia estimada de 10,4 millones; los datos más recientes sobre los resultados del tratamiento muestran una tasa mundial de éxitos terapéuticos del 83\%, similar a la de los últimos años (Organización Mundial de la Salud, 2017).

Es una enfermedad que no solo afecta la parte física de las personas sino trae consigo problemas morales, sociales y económicos. Por lo antes mencionado las personas que padecen de esta enfermedad, en muchos casos son rehuidos, aislados de los ambientes familiares y de trabajo.

La problemática social de esta enfermedad actualmente se centra en el riesgo de incumplimiento terapéutico y su posible influencia negativa tanto en la salud del paciente como en el control de la enfermedad desde el punto de vista de la salud pública. La complicación de la adherencia del tratamiento antituberculoso tiene connotaciones graves, como son el deterioro físico de la salud del paciente por la enfermedad, la posibilidad de estimular los mecanismos de resistencia bacteriana, la continuación de la propagación de la infección y la perpetuación de su existencia en la humanidad. En esta patología el paciente no puede alegar su autodeterminación a rehusar el tratamiento porque está exponiendo a las demás personas a contraer el bacilo, pero tampoco se puede forzar al paciente a cumplir sin tener en cuenta y respetar los principios éticos (Pozsik, 2013).

\footnotetext{
57 Vol. 5, núm. 1, enero 2019, pp. 54-68 María A. Cedeño-Ugalde; Fátima M. Figueroa-Cañarte; Jonathan R. Zambrano-Barre; Claudia V. Romero-Castro; Carmen V. Arias-Nazareno; Elio A. Santos-Andrade
} 
Son múltiples las razones para la no adherencia a un tratamiento, se ha considerado que la falta de observancia de los esquemas de tratamiento es la principal causa de falla terapéutica; no obstante, su cumplimiento puede cambiar en el tiempo por la percepción del paciente sobre su eficacia o deficiencia, por la toma simultánea de varios medicamentos, por la forma en que afecta el desempeño cotidiano, así como por factores económicos, laborales, socioculturales, ambientales, y los relacionados específicamente con el medicamento (Galeano \& Correa, 2005).

Otros factores que también predisponen a abandonar el tratamiento son la baja escolaridad, la información recibida sobre la enfermedad y los métodos para tratarla, así como el sistema de salud al que pertenece el paciente, la separación o disolución del núcleo familiar por temor al contagio y el hecho de convivir en un entorno familiar conflictivo (Culqui, Grijalva, Reategui, Cajo, \& Suarez, 2005).

El apoyo familiar interviene frente a los eventos que afectan la salud; al estar en contacto permanente con el paciente, el familiar participa de manera activa y de manera trascendentalmente para favorecer el cumplimiento del tratamiento, no solo farmacológico sino también los cambios en sus hábitos de vida. La familia es responsable de su adherencia al tratamiento, ya que muchas veces ellos se ven inmersos en un fuerte perjuicio o estigmatización social, donde la familia trata de ocultarlos, lo cual evidencia que su participación es primordial para llevar con éxito la adherencia al tratamiento.

El apoyo de la familia es el eje fundamental para motivar al paciente a tomar el tratamiento completo contra la Tuberculosis hasta su total restablecimiento, de acuerdo a las indicaciones del personal de salud y realizando los controles médicos respectivos (Fabelo, Iglesias, Bachir, Ramírez, \& Amador, 2013). Así también, el apoyo afectivo es esencial ya que permite acompañar al paciente 
en el curso de la enfermedad, evitando pensamientos de negatividad y baja autoestima. La

Tuberculosis Pulmonar se acompaña de un debilitamiento general, con poca energía y capacidad física para las actividades cotidianas. El estigma social causa un gran impacto en el paciente; es por ello, que se siente rechazado y excluido de la sociedad, deteriorando su autoestima (Contreras, 2012).

Los problemas de adherencia de los pacientes al tratamiento podrían manejarse de mejor manera conociendo el entorno familiar e identificando el apoyo que reciben de los mismos, estas variables según los estudios se encuentran relacionados, con el éxito del tratamiento. En la primera parte se abordan aspectos relevantes sobre el apoyo familiar y el rol de la Familia. En la segunda parte se tomó en consideración la adherencia al tratamiento y las razones para falta de adherencia.

Para finalizar, se sintetiza cómo la falta de apoyo familiar influye en la no adherencia al tratamiento de la Tuberculosis.

\section{Metodología.}

Se trata de una revisión narrativa-descriptiva, basada en el análisis de la literatura publicada en libros, artículos de revistas y análisis crítico del autor con el objetivo de integrar y describir el apoyo familiar en la adherencia al tratamiento de pacientes con tuberculosis.

\section{Desarrollo}

La Tuberculosis es una enfermedad infecciosa causada por la bacteria Mycobacterium tuberculosis, bacilo Gram positivo alcohol acidorresistente, el cual es transmitido de persona a persona por vía aérea de un paciente que tiene la enfermedad pulmonar activa (Organizacion

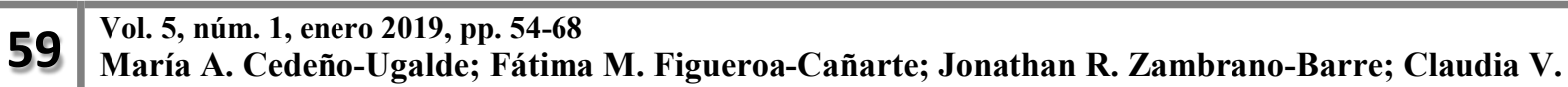
Romero-Castro; Carmen V. Arias-Nazareno; Elio A. Santos-Andrade
} 
Mundial de la Salud, 2014). La mayoría de los casos son de inicio insidioso y poco alarmante dependiendo de la virulencia del agente causal, la edad, el órgano afectado y el estado inmunológico del paciente, produciendo síntomas sistémicos (fiebre, sudoración nocturna profusa, pérdida de apetito y peso) y específicos del órgano (pulmonar, ganglionar) (2008).

El tratamiento de esta enfermedad se basa en la administración de medicamentos combinados durante al menos seis meses. Durante este lapso, el soporte social del paciente es importante, dado que mejora su adherencia al tratamiento y su calidad de vida (2011).

Palma (1993) menciona que la efectividad del tratamiento en los casos infecciosos de la enfermedad es el control de la Tuberculosis. El cumplimiento y terminación del tratamiento debe ser prioridad en cualquier programa; de hecho, algunos autores indican que si no es posible asegurar que el paciente tuberculoso complete el tratamiento, es preferible no iniciarlo dadas las graves consecuencias de la no adherencia, tanto en el ámbito individual, como colectivo. Así mismo el abandono del tratamiento constituye un importante problema para la Salud Pública

\section{Apoyo Familiar}

Se entiende por apoyo familiar, la presencia de la unidad familiar durante el proceso de enfermedad, discapacidad y asistencia de uno de sus miembros. Los integrantes de la familia deben ofrecer su apoyo emocional, mantener con el paciente una buena comunicación, participar en su cuidado y estar en contacto con otros miembros de la familia para informarles y demandarles ayuda, si fuera necesario. En relación con el apoyo familiar, toma especial protagonismo el rol de cuidador familiar (Rodríguez \& Pastor, 2000). 
Es aquí donde se integran el conjunto de actividades y estrategias socioeducativas de carácter grupal que promueven el desarrollo y bienestar de las familias, de tal forma que, atendiendo a la globalidad de las mismas, se pueda promover la educación en valores, fomentar hábitos de vida saludable, desarrollar las competencias parentales y facilitar la construcción de vínculos estables positivos entre los miembros de la familia (Meza, 2010).

Morguefile (2014) refiere que dentro de la formación del individuo en el transcurso de su vida, es importante el papel que juega la familia, ya que es una necesidad básica del hombre. Esta unidad de personalidades forma vínculos entre sí, interactuando dentro de los sistemas de emociones y necesidades, manteniendo una integridad social relacionada por fuertes afectos.

El clima social familiar se da en un ambiente de cohesión, expresivo, conflictivo, independiente, orientado a metas y cultura intelectual, recreando religiosidad, formación y control. Sus peculiaridades socio ambientales son paralelas a las relaciones interpersonales en la familia, con estructura básica y estable. (Espinoza, 2018)

Por lo tanto, el apoyo familiar interviene frente a los eventos que afectan la salud. Al estar en contacto permanente con el paciente, el familiar participa de manera activa para favorecer el cumplimiento del tratamiento, no solo farmacológico sino también en los cambios de sus hábitos de vida. González (2011) indica que la familia es responsable de su adherencia al tratamiento, ya que muchas veces son estigmatizados socialmente y la familia trata de ocultarlos, lo cual evidencia la importancia de la participación y el apoyo de la familia para llevar con éxito la adherencia al tratamiento. 
Achury y Pinilla (2016) indican que la familia es el eje fundamental para garantizar que el paciente tome el tratamiento completo contra la Tuberculosis hasta lograr su total restablecimiento, de acuerdo a las indicaciones del personal de salud y realizando los controles médicos respectivos. Así también, Romo (2010) menciona que el apoyo afectivo es esencial ya que permite que el paciente en el curso de la enfermedad, evite pensamientos negativos y de baja autoestima.

\section{Adherencia al tratamiento}

Es el cumplimiento estricto del tratamiento, que comprende el tratamiento farmacológico, las indicaciones de seguimiento y la consejería de enfermería, se obtendrá a través de una fícha de registro y se valorara como adherencia o no adherencia, también se aplicara una encuesta sobre escala de actitudes frente al tratamiento por tuberculosis y se valorara como actitud positiva o negativa, y por ultimo IMC y se valora como adecuado o inadecuado (Salas \& Chamizo, 2018).

El concepto de adherencia ha sido ampliamente discutido en la literatura por las disciplinas de cuidados de la salud, incluyendo enfermería, medicina, psicología, farmacia, terapia física y nutrición. Según la OMS (2004), la adherencia al tratamiento se define como el grado en que el paciente sigue las instrucciones médicas; esta definición incluye los conceptos de cumplimiento por parte del paciente del régimen terapéutico prescrito, además de persistencia estricta del mismo a lo largo del tiempo. Esto debe suponer la aceptación por parte del paciente, el compromiso para iniciar y seguir su tratamiento en las condiciones pautadas

La adherencia a corto y largo plazo es el resultado de un proceso complejo que se desarrolla a través de diferentes etapas: la aceptación del diagnóstico, la percepción de la necesidad de realizar el tratamiento de forma correcta, la motivación para hacerlo, la disposición y entrenamiento de

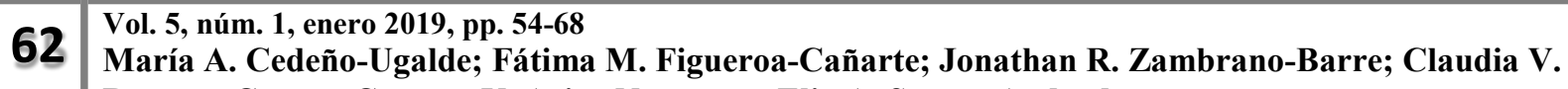
Romero-Castro; Carmen V. Arias-Nazareno; Elio A. Santos-Andrade
} 
habilidades para realizarlo, la capacidad de superar las barreras o dificultades que aparezcan, y el mantenimiento de los logros alcanzados con el paso del tiempo (Fernández, 2003).

Cáceres (2004) "El incumplimiento o falta de apego del paciente a la medicación implica graves consecuencias tanto para el enfermo como para la comunidad, debido a que el paciente no se cura y continúa la cadena de transmisión de la enfermedad". Adicionalmente, refiere que el Sistema de Tratamiento por Observación Directa (DOTS por sus siglas en inglés) refiere que la bacteria puede desarrollar resistencia a los medicamentos disponibles para el tratamiento, aumentando los costos del mismo y la mortalidad de los pacientes. Ortego, González y Álvarez (2001) mencionan que disminuye la eficacia de los programas nacionales al incrementar el riesgo de transmisión del bacilo y la probabilidad de resistencia bacteriana a los antibióticos, la cual predispone a una mayor frecuencia de recidivas y fracasos.

Las razones para falta de adherencia al tratamiento son:

- Desconocimiento de la enfermedad.

- Situación económica, aunque el medicamento es gratuito, tiene que desplazarse a un centro de salud para recibirlo, puede no tener dinero para el traslado.

- Efectos colaterales del fármaco, se siente peor tomando el medicamento que padeciendo la enfermedad.

- Falta de empatía del equipo de salud.

- Vergüenza de tener la enfermedad y no quiere que su entorno lo descubra.

- Falta de apoyo familiar, también por desconocimiento de la enfermedad. 
Cadena (2011) indica que La adherencia terapéutica implica una diversidad de conductas, por lo que ha sido considerada como un fenómeno múltiple y complejo. Al constituir una conducta de salud para su explicación se ha partido de los modelos de comportamientos de salud. Especialmente el modelo de creencias de salud ha sido el más utilizado y considerado adecuando para predecir el cumplimiento con la medicación, porque tienen en cuenta la motivación por la salud, la vulnerabilidad y gravedad percibida, la valoraron diferencial costos-beneficios y las claves para la acción.

Se considera un fenómeno complejo, entendiéndose como un comportamiento humano que está condicionado por múltiples factores de diversa naturaleza, entre los cuales se menciona: los relacionados con la interacción de la persona y profesional de salud, el régimen terapéutico en sí mismo, las características de la enfermedad y los aspectos psicosociales de la persona.

Para la rama de la salud proporcionar apoyo, educación, y cuidado, responde a las necesidades bien sea en situaciones permanentes o en situación transitorias. Los profesionales que proveen soporte formal pueden contribuir a reforzar y adecuar los recursos familiares u otros apoyos de la comunidad.

Las redes formales e informales son recursos valiosos a la hora de brindar apoyo, sobre todo para pacientes crónicos y se puede concluir que el tamaño de la red social es un indicador del apoyo. La calidad y la disponibilidad de personas que conforman la red social contribuyen al éxito del apoyo para las personas que lo requieren. Es necesario que la enfermera valore aspectos tales como depresión, riesgo de lesiones y comportamientos de riesgo de los pacientes para poder intervenir de forma concordante con la problemática (Pérez, 2012). 
El abandono del tratamiento antituberculoso, tiene consecuencias graves como son: el deterioro de la salud del paciente, estimular los mecanismos de resistencia bacteriana predisponiendo al fracaso del tratamiento, la propagación de la infección debido a que el paciente no se cura y continua la cadena de transmisión de enfermedad, por lo tanto la perpetuación de la existencia de esta enfermedad en la humanidad. La OMS considera que la resistencia del bacilo a los fármacos aumenta, tanto el costo como la toxicidad de los medicamentos y la mortalidad de las personas que la desarrollan (2014).

Algunos estudios efectuados respecto a la no adherencia al tratamiento antituberculoso, destacan la existencia de múltiples factores; entre los cuales se encuentran los relacionados al tratamiento que recibe el paciente, algunos vinculados a la organización y funcionamiento de los servicios de salud y otros de orden socio económico cultural del paciente (Orozco, 2009).

En cuanto a los factores relacionados al tratamiento antituberculoso al que se acoge el paciente. Gelband (2000), demostró que la duración del tratamiento está asociada a la pobre adherencia y fue relacionada como el obstáculo más difícil a vencer para lograr la adherencia, así mismo Homedes (2004) comprueba que la cantidad de medicamentos que el paciente tiene que tomar es inversamente proporcional a la adherencia al tratamiento.

Respecto a los factores de orden socio económico y cultural, diversos estudios como el de Sumartojo (1993) explican que contar con una red de apoyo social se asocia como factor protector para adherencia al tratamiento, así como la conformación de clubes como mecanismo de apoyo social ha demostrado mejorar la adherencia al tratamiento.

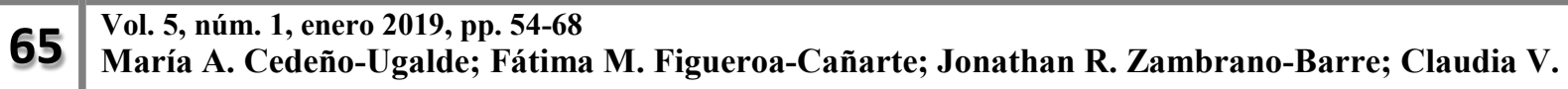
Romero-Castro; Carmen V. Arias-Nazareno; Elio A. Santos-Andrade
} 
Conflicto de Intereses

Los autores declaran que no existen conflictos de intereses.

\section{Declaración de contribución}

Todos los autores participaron en la búsqueda de información y en la redacción del artículo.

\section{Conclusiones.}

Podemos finalizar, considerando que la tuberculosis no es una enfermedad individual, por su alto contagio se requiere conocimiento sobre la infección y como prevenirla a nivel familiar y en las comunidades, para apoyar en el tratamiento con disminución de la resistencia a tratamiento y la disminución de la progresión de enfermedad y su contagio.

El punto de vista del paciente, la familia y su importancia en la adherencia al tratamiento, que habitualmente no se ha considerado en investigaciones sobre tuberculosis en el Ecuador, puede resultar muy útil para ajustar la oferta de servicios de salud y el control de la enfermedad, toda esta problemática se convierte en un factor que influye en la adherencia del tratamiento, en la falta de regularidad y desde luego deteriora aún más la salud del usuario con tuberculosis.

La educación debe ser impartida también a su entorno, la familia, para que brinde el apoyo emocional necesario para cumplir el tratamiento. Si existe una relación significativa y moderada entre ambas variables, nivel del apoyo familiar a paciente y el nivel de adherencia al tratamiento de pacientes con tuberculosis, a mayor apoyo familiar mayor será la adherencia al tratamiento de los pacientes con tuberculosis. 


\section{Bibliografía.}

Achury, D., \& Pinilla, M. (2016). La comunicación con la familia del paciente que se encuentra al final de la vida. . Enfermería universitaria, 134-141.

Cadena, ,. F. (2011). Eficacia de una Intervención Promotora de Salud en la Adherencia al Tratamiento $y$ Estilo de Vida en Enfermos de Tuberculosis Pulmonar. Obtenido de http://rua.ua.es/dspace/bitstream/10045/24913/1/Tesis\%20Francisco\%2 0Cadena\%20Santos.pdf

Contreras, A. (Febrero de 2012). Percepción de apoyo social por pacientes con enfermedad alcohólica en fase de recuperación. Rev. Hosp. Psiquiatrico de la Habana, 13(2).

Culqui, D., Grijalva, C., Reategui, S., Cajo, J., \& Suarez, L. (2005). Recuperado el 27 de Septiembre de 2018, de Factores pronosticos del abandono del tratamiento antituberculoso en una region endemica del Peru: http://dx.doi.org/10.1590/S1020-49892005000600003

Espinoza, E. (2018). Clima social familiar y autoestima de los Pacientes con tuberculosis pulmonar que Asisten al Centro De Salud Caqueta Rimac. Tesis para optar el título de enfermería. Lima Perú.

Fabelo, J., Iglesias, S., Bachir, S., Ramírez, A., \& Amador, J. (Abril de 2013). Factores que influyen en la adherencia a tratamientos farmacológicos y no farmacológicos en los pacientes inscritos en el Programa de Control de Tuberculosis de la Unidad Básica de Atención de COOMEVA. Revista Cielo, 26(2).

Fernández, A. (2003). DOTS, un tratamiento que puede curar a los pacientes. El Universo, 18.

Galeano, S., \& Correa, J. (2005). Adherencia al tratamiento, implicaciones de la no-adherencia. Acta Medica Colombia, (págs. 268-273). Colombia.

Gelband. (2000). Abandono de tratamiento antituberculoso. Med. Colombia, 136.

Gonzales, C. P. (2011). El apoyo familiar en la adherencia al tratamiento nutricional del paciente con diabetes mellitus tipo 2 (DM2). . Waxapa, 102-106.

Homedes, M. (2004). Perfiles de incumplimiento de terapia antituberculoso. Clinica Medica, 89.

Meza, M. (2010). El proyecto educativo integral comunitario: un instrumento para la promoción de la participación del docente, . RevinPost, 129-142.

Ministerio de Salud Pública del Ecuador. (2017). Plan Estratégico Nacional para la prevención y control de la tuberculosis en Ecuador.

Ministerio de Salud Pública del Ecuador. (2017). Sistema de Información de TB.

Ministerio de Salud-Lima, Perú. (2011). Respira vida, juntos contra la tuberculosis. Obtenido de Respira vida, juntos contra la tuberculosis.

Moreno, F. M. (Agosto de 2005). Importancia de los modelos conceptuales y teorías de enfermería: experiencia de la Facultad de Enfermería de la Universidad de La Sabana. Aquichan. 
Morguefile, C. (2014). Importancia de la familia en la vida de las personas. Obtenido de https://rpp.pe/lima/actualidad/importancia-de-la-familia-en-la-vida-de-las-personas-noticia668100

Organizacion Mundial de la Salud. (2004). Adherencia a los tratamientos a largo plazo. Pruebas para la acción. Obtenido de http://www.paho.org/Spanish/AD/DPC/NC/adherencia-largo-plazo.pdf

Organizacion Mundial de la Salud. (2014). Recuperado el 25 de Septiembre de 2018, de ¿Que es la tuberculosis y como se trata?: http://www.who.int/features/qa/08/es/.

Organización Mundial de la Salud. (2017). Informe mundial sobre la tuberculosis 2017.

Organización Mundial de la Salud. (2018). Recuperado el 28 de Septiembre de 2018, de Tuberculosis: http://www.who.int/es/news-room/fact-sheets/detail/tuberculosis

Orozco, P. (2009). Factores de riesgo para el abandono del tratamiento antituberculoso. Rev. Medicas. . Col.Colombia, 34-39.

Ortego, M., Gonzalez, S., \& Álvarez, M. (2001). La adherencia al tratamiento. Open Course Wour, 23441. .

Palma, B. O. (1993). El abandono del tratamiento en tuberculosis.Realidad actual y perspectivas futuras. Arq. Tuberc, XLIV(1).

Pérez, B. G. (2012). El cuidado de la enfermería en familia: un reto para el siglo XXI. Aquichan, 23-31.

Pozsik, C. (2013). Compliance with tuberculosis therapy. Med. Clin. North AM.

Rae, J. (Marzo de 2018). América Latina y el Caribe pueden acabar con la tuberculosis, pero falta compromiso. Noticias ONU.

Rodríguez, J., \& Pastor, M. A. (2000). Afrontamiento, apoyo social, calidad de vida y enfermedad. . Psicothema, , 349-372.

Salas, P., \& Chamizo, H. (2018). Determinantes sociales de la adherencia al tratamiento de la tuberculosis. Obtenido de Discusion desde la perspectiva heuristica del riesgo: http://www.scielo.sa.cr/scielo.php?pid=S140912592002000100006\&sc ript=sci_arttext\&tlng=e

Sociedad Españñola de Neumología y cirugía torácica. (2008). Recuperado el 2018 de Septiembre de 25, de Normativa SEPAR: Diagnóstico y Tratamiento de la Tuberculosis.: http://issuu.com/separ/docs/normativa_050/3

Villegas, C. M., Merino, C. M., \& Jaime Hernandez, N. (2018). Fundamentos Teóricos y prácticos de enfermería. Jipijapa: Mawil Publicaciones. 